\title{
Toxicokinetic Interaction between Hepatic Disposition and Pulmonary Bioactivation of Inhaled Naphthalene Studied Using Cyp2abfgs-Null and CYP2A13/2F1-Humanized Mice with Deficient Hepatic Cytochrome P450 Activity ${ }^{\mathbb{S}}$
}

\author{
Nataliia Kovalchuk, Qing-Yu Zhang, Jacklyn Kelty, Laura Van Winkle, and Xinxin Ding
}

Department of Pharmacology and Toxicology, College of Pharmacy, University of Arizona, Tucson, Arizona (N.K., Q.-Y.Z., X.D.); Wadsworth Center, New York State Department of Health, and School of Public Health, State University of New York, Albany, New York (N.K., Q.-Y.Z.); Center for Health and the Environment, Department of Anatomy, Physiology and Cell Biology, School of Veterinary Medicine, University of California, Davis, California (J.K., L.V.W.); and College of Nanoscale Science, State University of New York Polytechnic Institute, Albany, New York (X.D.)

Received August 9, 2019; accepted October 1, 2019

\section{ABSTRACT}

Previous studies using Cyp2abfgs-null (lacking all genes of the Cyp2a, 2b, 2f, 2g, and 2s subfamilies), CYP2A13/2F1-humanized, and liver-Cpr-null (LCN) mice showed that although hepatic cytochrome P450 (P450) enzymes are essential for systemic clearance of inhaled naphthalene (a possible human carcinogen), both hepatic and extrahepatic P450 enzymes may contribute to naphthaleneinduced lung toxicity via bioactivation. Herein, we aimed to further understand the toxicokinetics of inhaled naphthalene in order to provide a basis for predicting the effects of variations in rates of xenobiotic disposition on the extent of target tissue bioactivation. We assessed the impact of a hepatic deficit in naphthalene metabolism on the toxicokinetics of inhaled naphthalene using newly generated Cyp2abfgs-null-and-LCN and CYP2A13/2F1humanized-and-LCN mice. We determined plasma, lung, and liver levels of naphthalene and naphthalene-glutathione conjugate, a biomarker of naphthalene bioactivation, over time after naphthalene inhalation. We found that the loss of hepatic naphthalene metabolism severely decreased naphthalene systemic clearance and caused naphthalene to accumulate in the liver and other tissues. Naphthalene release from tissue, as evidenced by the continued increase in plasma naphthalene levels after termination of active inhalation exposure, was accompanied by prolonged bioactivation of naphthalene in the lung. In addition, transgenic expression of human CYP2A13/2F1 in the respiratory tract caused a reduction in plasma naphthalene levels (by $40 \%$, relative to Cyp2abfgs-null-and-LCN mice) and corresponding decreases in naphthalene-glutathione levels in the lung in mice with hepatic P450 deficiency, despite the increase in local naphthalene-bioactivating P450 activity. Thus, the bioavailability of naphthalene in the target tissue has a significant effect on the extent of naphthalene bioactivation in the lung.

\section{SIGNIFICANCE STATEMENT}

In this study, we report several novel findings related to the toxicokinetics of inhaled naphthalene, the ability of which to cause lung carcinogenesis in humans is a current topic for risk assessment. We show the accumulation of naphthalene in the liver and lung in mice with compromised hepatic cytochrome P450 (P450) activity; the ability of tissue-stored naphthalene to redistribute to the circulation after termination of active inhalation exposure, prolonging exposure of target tissues to naphthalene; and the ability of nonCYP2ABFGS enzymes of the lung to bioactivate naphthalene. These results suggest potentially large effects of deficiencies in hepatic P450 activity on naphthalene tissue burden and bioactivation in human lungs.

\section{Introduction}

Naphthalene (NA) is present ubiquitously in the environment (Witschi et al., 1997; Kakareka and Kukharchyk, 2003). High exposure to NA occurs in occupational settings (Preuss et al., 2003; Chao et al.,

This work was supported in part by the National Institutes of Health National Institute of Environmental Health Sciences [Grants R-01 ES020867, P30 ES023513, and P30 ES006694] and the National Institutes of Health National Heart, Lung, and Blood Institute [Grant T32-HL007013 (to J.K.)].

https://doi.org/10.1124/dmd.119.088930.

S This article has supplemental material available at dmd.aspetjournals.org.
2006). Urinary naphthol levels were elevated in the postshift, compared with preshift, period among exposed workers (Serdar et al., 2004; McClean et al., 2012; Merchant-Borna et al., 2012), providing evidence for NA metabolism in humans. NA metabolism by human liver microsomes has also been documented (Tingle et al., 1993; Wilson et al., 1996). However, the question remains whether NA-induced respiratory tract toxicity, as has been found in animal models (Warren et al., 1982; Abdo et al., 1992, 2001), occurs in the human respiratory tract. There are no clear-cut human epidemiologic data on the long-term effects of NA exposure because human NA exposures are often as a part of mixtures (e.g., cigarette smoke) (Griego et al., 2008).

ABBREVIATIONS: AP, acetaminophen; AUC, area under the curve; CAS, Chemical Abstract Service; Cpr, cytochrome P450 reductase; GSH, glutathione; LCN, liver-Cpr-null; NA, naphthalene; P450, cytochrome P450; WT, wild type. 
The toxic effects of NA are dependent on cytochrome P450 (P450)-mediated bioactivation via NA-oxide formation (Buckpitt et al., 2002). CYP2F2, expressed predominantly in mouse airways and in the liver, has the lowest $K_{\mathrm{m}}$ among P450 enzymes studied for NA bioactivation (Shultz et al., 1999) and plays a dominant role in NA-induced pulmonary damage (Verschoyle et al., 1997; Genter et al., 2006; Li et al., 2011). In contrast, CYP2A5, abundantly expressed in the mouse nasal olfactory mucosa, lung, and liver, is the main contributor to NA-induced nasal toxicity (Hu et al., 2014). Human CYP2F1 and CYP2A13 are mainly expressed in the respiratory tract in humans, with negligible expression in other tissues (Su et al., 2000; Carr et al., 2003; Weems and Yost, 2010). Metabolism of inhaled NA by respiratory tract-expressed transgenic CYP2F1 and CYP2A13 resulted in cytotoxicity in lung airway epithelia and nasal olfactory mucosa in CYP2A13/2F1-humanized mice, highlighting the potential impact of the human enzymes on NA metabolism and respiratory tract toxicity (Li et al., 2017).

Several P450 isoforms with the ability to metabolize NA are also identified in the human liver, including CYP1A2, CYP2A6, CYP3A4, and CYP2D6 (Cho et al., 2006; Shimada et al., 2016). Hepatic P450mediated NA metabolism has a major impact on the in vivo disposition of inhaled or intraperitoneally administered NA, as indicated by studies comparing wild type (WT) and liver-Cpr-null (LCN) mice (Li et al., 2011; Kovalchuk et al., 2017). However, both hepatic and extrahepatic P450-mediated NA metabolism also contributed to toxicity in lung airways after inhalation exposure to NA at occupationally relevant concentrations (Kovalchuk et al., 2017). These seemingly opposing actions of hepatic P450-mediated NA metabolism make it complicated to predict consequences of alterations in hepatic NA metabolism on risks of lung toxicity. It is also unclear to what degree circulating NA can influence the extent of target tissue NA bioactivation in an inhalation exposure model, where the lung is directly exposed to NA in the inhaled air, or whether extrahepatic organ NA metabolism contributes significantly to systemic disposition of NA when hepatic P450 activity toward NA is deficient. Thus, a better understanding of the relationships between hepatic P450 activity, which may vary due to induction, inhibition, disease conditions, or genetic polymorphism, and the toxicokinetics of NA after inhalation exposure might help with prediction of the risks of NA toxicity in individuals with compromised hepatic $\mathrm{P} 450$ activity.

In the current study, we aimed to study the impact of a hepatic deficit in NA metabolism on the toxicokinetics of inhaled NA. The hepatic deficit was modeled using LCN mice, which undergo tissue-specific deletion of the cytochrome $\mathrm{P} 450$ reductase $(\mathrm{CPr})$ gene in hepatocytes, resulting in tissue-specific ablation of nearly all microsomal $\mathrm{P} 450$ activity in the liver (Gu et al., 2003). We crossbred the LCN mice with mice that are "humanized" by having a much lower overall rate of NA metabolism in the lung (relative to WT mice), because of germline (whole body) deletion of the Cyp2abfgs gene cluster (including all genes in the Cyp2a,Cyp2b,Cyp2f,Cyp2g, and Cyp2s subfamilies) (Li et al., 2014), and by expressing human CYP2A13 and CYP2F1 (Wei et al., 2012). We exposed CYP2A13/2F1-humanized and Cyp2abfgs-null mice with either normal or compromised hepatic P450 activity (referred to hereafter as Cyp2abfgs-null-and-LCN mice and CYP2A13/2F1-humanizedand-LCN mice, respectively) to inhaled NA at an occupationally relevant concentration $(10 \mathrm{ppm})$. We then determined the levels of NA and NA-glutathione (GSH) conjugate, a biomarker of P450catalyzed NA bioactivation via NA-oxide formation, in plasma, liver, and lung at various times after termination of inhalation exposure. The toxicokinetic data from these various mouse strains were compared to identify the roles of hepatic and respiratory tract P450 enzymes in NA distribution, bioactivation, and disposition.

\section{Materials and Methods}

Chemicals and Reagents. NA [Chemical Abstract Service (CAS) number 91-20-3, purity 99\%], NA- $d_{8}$ [CAS number $1146-65-2$, purity $99 \%$ ], GSH [CAS number 70-18-8, purity $\geq 98.0 \%$ ], and NADPH [CAS number 100929$71-3$, purity $\geq 95.0 \%$ ] were purchased from Sigma-Aldrich (St. Louis, MO). Acetaminophen (AP)-GSH was purchased from Toronto Research Chemicals (Toronto, ON, Canada). NA-GSH standard as a mixture of all four stereoisomers was a generous gift from Drs. Alan R. Buckpitt and Dexter Morin (University of California, Davis, CA) and was prepared as previously described (Richieri and Buckpitt, 1987). All solvents (dichloromethane, formic acid, methanol, and water) were of analytical grade (Fisher Scientific, Houston, TX).

Mouse Breeding and Characterization. Cyp2abfgs ${ }^{-/-}$(Cyp2abfgs-null), CYP2A13/2F1-TG ${ }^{+/-} /$Cyp2abfgs $^{-1-}$ (CYP2A13/2F1-humanized), and LCN (albumin-Cre/ $\mathrm{Cp} r^{\text {lox/lox}}$ ) mice (male and female, all on the C57BL/6 background) were obtained from breeding stocks maintained at the Wadsworth Center. Progeny of Cyp2abfgs-null and LCN intercrosses were further intercrossed with CYP2A13/2F1-humanized mice to generate CYP2A13/2F1-humanized-andLCN mice and Cyp2abfgs-null-and-LCN mice, respectively. A detailed breeding scheme is presented in Fig. 1A. All pups were genotyped using tail DNA for the presence of the Cre transgene, the loxP sites, and the Cyp $2 \mathrm{f}^{+}$, Cyp $2 a b f g s^{-}$, and the $\mathrm{CYP} 2 \mathrm{~F} 1^{+}$alleles. For genotype analysis, PCR was carried out for 35 cycles with an annealing temperature at $60^{\circ} \mathrm{C}$ for 30 seconds and extension at $72^{\circ} \mathrm{C}$ for 1 minute for each cycle. PCR primers for, and representative results of, the genotyped alleles are presented in Supplemental Table 1 and Fig. 1B, respectively. PCR products were separated by electrophoresis in agarose gels and visualized after staining with ethidium bromide.

Serum levels of albumin, blood urea nitrogen, total bilirubin, alkaline phosphatase, alanine aminotransferase, and cholesterol were estimated for naïve Cyp2abfgs-null, CYP2A13/2F1-humanized, Cyp2abfgs-null-and-LCN, and CYP2A13/2F1-humanized-and-LCN male mice with a VETSCAN VS2 Chemistry Analyzer, using the Mammalian Liver Profile rotors (Abaxis, Union City, CA). A $100-\mu 1$ aliquot of serum from each mouse was analyzed.

Assay for NA Bioactivation In Vitro. The rate of NA-GSH formation was measured according to a previously described protocol (Shultz et al., 1999). Microsomes were prepared from individual livers, pooled lungs, or pooled olfactory mucosa of male 2- to 3-month-old mice. Reaction mixtures contained $50 \mathrm{mM}$ phosphate buffer (pH 7.4), NA (100 $\mu \mathrm{M}$, added in $1 \mu 1$ methanol), $10 \mathrm{mM}$ GSH, $1 \mathrm{mM} \mathrm{NADPH}$, and different concentrations of microsomal proteins $(0.2 \mathrm{mg} / \mathrm{ml}$ for liver, $0.25 \mathrm{mg} / \mathrm{ml}$ for olfactory mucosa, and $1 \mathrm{mg} / \mathrm{ml}$ for lung) in a final volume of $0.1 \mathrm{ml}$. The reactions were carried out in tightly capped glass tubes (2-dram vials) at $37^{\circ} \mathrm{C}$ for 10 minutes for liver, 30 minutes for olfactory mucosa, and 60 minutes for lung microsomal proteins. The reaction was quenched by transferring the incubation tubes to an ice bath and adding $0.2 \mathrm{ml}$ ice-cold methanol containing the internal standard AP-GSH to the uncapped tubes. The resultant mixtures were centrifuged twice to remove precipitated proteins and aliquots of supernatants were injected into an ultra-performance liquid chromatography-tandem mass spectrometry system for analysis, as previously described (Kovalchuk et al., 2017). NADPH, GSH, or NA were omitted, or boiled microsomes were used, in negative control incubations.

Animal Exposure. All animal studies were approved by the Wadsworth Center Institutional Animal Care and Use Committee. Two- to three-month-old male mice were used for the study. NA inhalation exposure settings were the same as described in detail elsewhere (Kovalchuk et al., 2017). Mice were nose-only exposed to a single 4-hour session of $10 \mathrm{ppm}$ NA (U.S. Occupational Safety and Health Administration-established exposure limit for occupational settings) or high-efficiency particulate air-filtered air (sham-exposure control) in an OroNasal and Respiratory Exposure System ( $\mathrm{CH}$ Technologies, Westwood, NJ). Male mice were chosen for the study, to be consistent with previous studies on NA lung toxicity (Kovalchuk et al., 2017; Li et al., 2017).

For plasma toxicokinetics studies, blood samples $(20-25 \mu \mathrm{l})$ were collected via tail vein from each mouse ( $n=3$ to 4 per each genotype) at multiple time points $(0,1,4,8$, and 20 hours) after termination of NA exposure. For tissue toxicokinetics studies, mice of all four genotypes were exposed simultaneously for each time point ( $n=3-5$ per each genotype for each time point), and different mice were used for different time points. Tissues (lung and liver) were collected at $0,2,6$, or 20 hours postexposure, quick-frozen on dry ice, and stored in sealed tubes at $-80^{\circ} \mathrm{C}$ until analysis. 


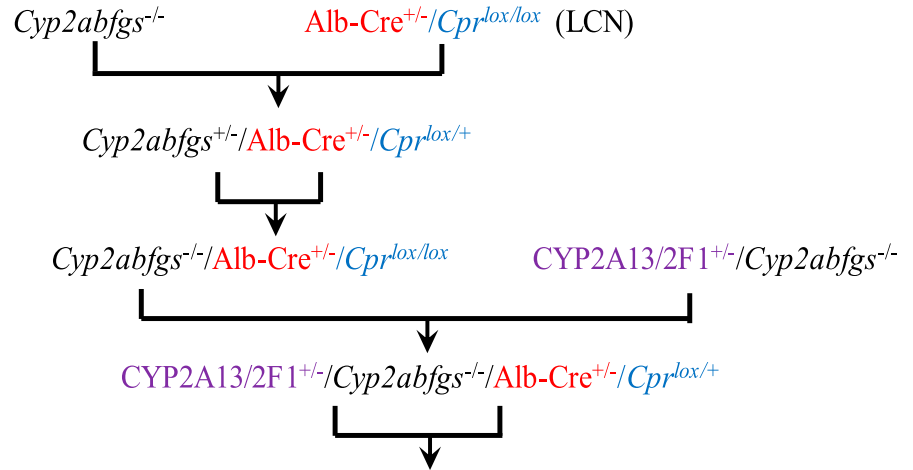

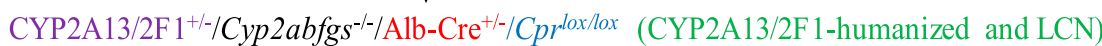

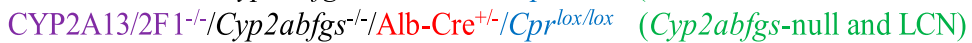

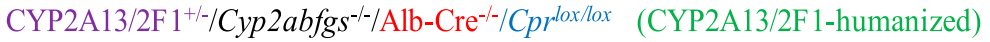

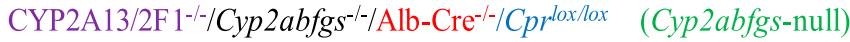

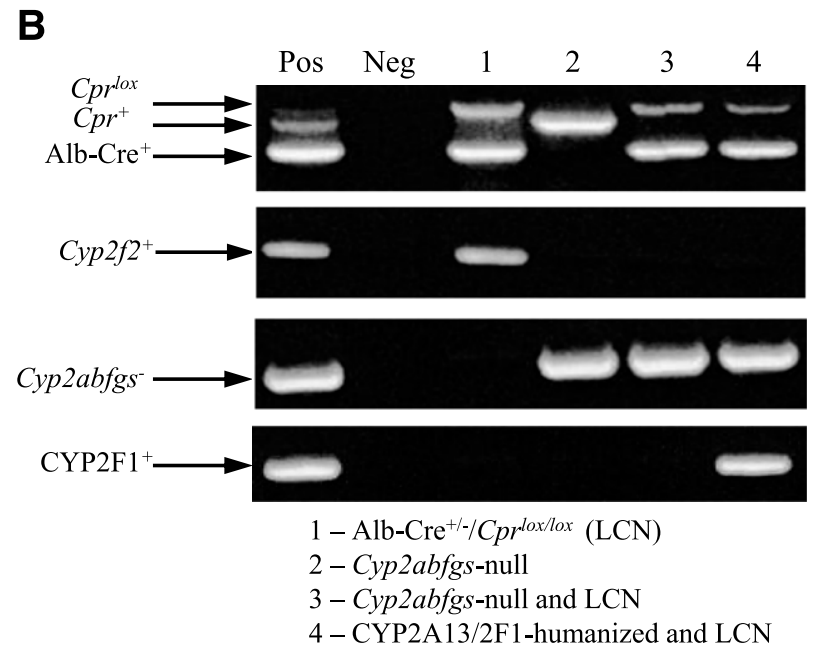

Fig. 1. Production and analysis of mouse models used in this study. (A) Breeding scheme for generating CYP2A13/2F1-humanized mice with either normal or abolished hepatic CPR expression. The original Cyp2abfgs ${ }^{-1-}$, Alb-Cre ${ }^{+1-} / \mathrm{Cpr}^{\text {lox/lox }}$ (LCN), and CYP2A13/2F1 ${ }^{+/-} /$Cyp $2 a b f g s^{-/-}$ (CYP2A13/2F1-humanized) strains were intercrossed to yield the four desired genotypes for this study. All mice are on the C57BL/6 genetic background. (B) Representative results of PCRbased genotyping to identify the presence of various alleles, including Cyp2abfgs ${ }^{-}$, Alb-Cre ${ }^{+}$, $\mathrm{Cpr}^{\text {lox }}, \mathrm{Cpr}^{+}, \quad \mathrm{Cyp} 2 \mathrm{f2}^{+}$(representing the Cyp2abfgs gene cluster), and $\mathrm{CYP}_{2} \mathrm{FI}^{+}$(representing both CYP2A13 and 2F1). The PCR primers used for the genotyping methods are listed in Supplemental Table 1. Positive controls (Pos) were derived from genomic DNA from $A l b$ $\mathrm{Cre}^{+/-} \mathrm{Cpr}^{\text {lox/+ }}$ mice for Alb-Cre ${ }^{+}, \mathrm{Cpr}^{+}, \mathrm{Cpr}^{\text {lox }}$, and Cyp2f2 or from CYP2A13/2F1-TG ${ }^{+-}$/ Cyp2abfgs $^{-1-}$ mice for Cyp2abfgs ${ }^{-}$and $\mathrm{CYP} 2 \mathrm{Fl}^{+} .{ }^{+}$Negative controls (Neg) were derived from reactions without an added DNA template. PCR products were analyzed and visualized as described in the Materials and Methods.
Detection of NA and NA-GSH. For NA detection, plasma (10 $\mu \mathrm{l})$ or lung homogenates $(50 \mu \mathrm{l})$ were spiked with NA- $d_{8}$ and extracted with dichloromethane (100 $\mu \mathrm{l}$ for plasma and $110 \mu \mathrm{l}$ for tissue) as previously described (Kovalchuk et al., 2017). Gas chromatography-mass spectrometry detection of NA $(\mathrm{m} / z, 128)$ and NA- $d_{8}(\mathrm{~m} / \mathrm{z}$ 136) was carried out using an Agilent Technologies model 5975 C inert XL EI/CI mass spectrometer (Agilent, Santa Clara, CA), which was interfaced with an Agilent model 7890 A gas chromatography system and operated in the selected ion monitoring mode using electron impact ionization. The extract $(1 \mu \mathrm{l})$ was injected in splitless mode, with the inlet at $260^{\circ} \mathrm{C}$. Analytes were separated on a Restek Rxi-5ms column ( $30 \mathrm{~m} \times 0.25 \mathrm{~mm}$; $0.25 \mu \mathrm{m}$; Restek, Bellefonte, PA) under a constant flow ( $1 \mathrm{ml} / \mathrm{min}$ ) of helium (Li et al., 2011). Blank plasma and blank tissue homogenate from naïve mice were spiked with freshly prepared NA standards $(5-500 \mathrm{ng} / \mathrm{ml})$ and NA- $d_{8}(5 \mathrm{ng}$ in $10 \mu$ l methanol). Ratios of detected NA and NA- $d_{8}$ were used to plot matrix-specific calibration curves and quantify NA in samples. The assay variability (relative S.D.) did not exceed $15 \%$; the limit for NA quantification (signal-to-noise ratio $>10$ ) was 5 pg on column.

For NA-GSH detection, reaction mixtures (100 $\mu \mathrm{l})$, plasma $(10 \mu \mathrm{l})$, or tissue homogenates $(50 \mu \mathrm{l})$ were spiked with internal standard AP-GSH and mixed with methanol (200, 80, and $90 \mu \mathrm{l}$, respectively) for protein precipitation, as described previously (Kovalchuk et al., 2017). NA-GSH and AP-GSH were separated at room temperature on an Agilent Eclipse Plus C18 column $(2.1 \times 50 \mathrm{~mm} ; 1.8 \mu \mathrm{m})$ at a flow rate of $0.2 \mathrm{ml} / \mathrm{min}$ with a mobile phase as previously described (Li et al., 2011), using an Agilent model 1290 Infinity Series ultra-performance liquid chromatography system. Analyte ions were detected using an AB-SCIEX model 6500 Q-Trap mass spectrometer (AB-SCIEX, Framingham, MA) in the positive ion mode using multiple reaction monitoring scanning. The transitions were $\mathrm{m} / \mathrm{z}$ $452 / 306$ and $m / z, 452 / 288$ (confirmatory) for NA-GSH quantification and $m / z$ 457/ 328 for AP-GSH quantification.

Matrix-matched calibration curves were prepared over the range of 5-227 ng/ml NA-GSH. AP-GSH (0.55 ng in $10 \mu \mathrm{l}$ methanol) was added to each sample. The assay variability (relative S.D.) did not exceed $15 \%$. The limit for NA-GSH quantification (signal-to-noise ratio $>10$ ) was $10 \mathrm{pg}$ on column.

Other Methods, Calculations and Statistical Analyses. The bicinchoninic acid assay was used to quantify total protein concentration (Pierce Chemical, Rockford, IL) using bovine serum albumin as a standard. Pharmacokinetic parameters, including the area under the curve (AUC), elimination half-life, and clearance, were calculated using WinNonlin software (Pharsight, Mountain View, CA), with the use of a noncompartmental model. Statistical significance of differences among study groups in various parameters was analyzed by one-way or two-way ANOVA, followed by the Bonferroni test for multiple comparisons, using GraphPad Prism software (GraphPad, San Diego, CA).

\section{Results}

Generation and Characterization of CYP2A13/2F1-Humanized-and-LCN Mice and Cyp2abfgs-Null-and-LCN Mice. Cyp2abfgs-null-and-LCN and CYP2A13/2F1-humanized-and-LCN mice were progeny of intercrosses between ${\text { Cyp } 2 a b f g s^{-1-}}($ Cyp $2 a b f g s-$ null), LCN, and CYP2A13/2F1-TG ${ }^{+/-} /$Cyp $2 a b f g s^{-/-}$(CYP2A13/2F1humanized) mice (Fig. 1A). In Cyp2abfgs-null-and-LCN and 
TABLE 1

Metabolic activity of nasal mucosa, lung, and liver microsomes of CYP2A13/2F1-humanized-and-LCN and Cyp2abfgs-null-and-LCN mice in vitro

Rates of NA-GSH formation were determined as described in the Materials and Methods. Values represent means \pm S.D. $(n=3$ to 4$)$.

\begin{tabular}{|c|c|c|c|c|c|}
\hline \multirow{2}{*}{ Tissue } & \multicolumn{5}{|c|}{ Rate of NA-GSH formation } \\
\hline & CYP2A13/2F1-humanized-and-LCN & Cyp2abfgs-null-and-LCN & CYP2A13/2F1-humanized $^{a}$ & Cyp2abfgs-null ${ }^{a}$ & $\mathrm{LCN}^{b}$ \\
\hline & \multicolumn{5}{|c|}{$\mathrm{pmol} / \mathrm{min}$ per mg microsomal protein } \\
\hline Nasal mucosa & $801 \pm 77$ & $206 \pm 6^{c}$ & $964 \pm 59$ & $118 \pm 12$ & N.A. \\
\hline Lung & $19.9 \pm 4.9$ & $11.9 \pm 0.5^{d}$ & $23.1 \pm 3.2$ & $8.1 \pm 0.5$ & $1120 \pm 198$ \\
\hline Liver & $76.9 \pm 13.5$ & $94.7 \pm 26.3$ & N.A. & N.A. & $83.7 \pm 8.7$ \\
\hline
\end{tabular}

Reaction mixtures contained $50 \mathrm{mM}$ phosphate buffer ( $\mathrm{pH}$ 7.4), $10 \mathrm{mM} \mathrm{GSH}, 1 \mathrm{mM}$ NADPH, various concentrations of microsomal proteins $(0.2 \mathrm{mg} / \mathrm{ml}$ fo individual livers, $0.25 \mathrm{mg} / \mathrm{ml}$ for pooled olfactory mucosa, and $1 \mathrm{mg} / \mathrm{ml}$ for pooled lung), and $100 \mu \mathrm{M} \mathrm{NA}$. Statistical analysis was performed using two-way ANOVA followed by the Bonferroni test for multiple comparisons. Previously reported data for LCN, Cyp2abfgs-null, and CYP2A13/2F1-humanized mice (Kovalchuk et al., 2017; Li et al., 2017) are shown for comparison. N.A., not analyzed.

${ }^{a}$ Extracted from Figure 1 of $\mathrm{Li}$ et al. (2017) for rates at $100 \mu \mathrm{M}$ NA.

${ }^{b}$ From original data used for kinetic analysis in Table 1 of Kovalchuk et al. (2017) for rates at $100 \mu \mathrm{M}$ NA.

${ }^{c} P<0.0001$, compared with CYP2A13/2F1-humanized-and-LCN mice.

${ }^{d} P<0.05$, compared with CYP2A13/2F1-humanized-and-LCN mice.

CYP2A13/2F1-humanized-and-LCN mice, the expression of Cre recombinase, driven by the rat albumin promoter, results in hepatocyte-specific deletion of the $C p r$ gene sequence between exons 3 and 15 (Wu et al., 2003) and, as has been shown previously (Gu et al., 2003), significantly lowered levels of CPR protein expression in the whole liver (Supplemental Fig. 1).

Cyp2abfgs-null-and-LCN and CYP2A13/2F1-humanized-and-LCN mice are normal in growth, development, general appearance, daily activity, and reproduction. Body weights and weights of the lung, kidney, brain, and heart of 2-month-old Cyp2abfgs-null-and-LCN and CYP2A13/2F1-humanized-and-LCN mice were unchanged compared with those of Cyp2abfgs-null and CYP2A13/2F1-humanized mice (Supplemental Table 2). However, enlargement of the liver was observed in the Cyp2abfgs-null-and-LCN and CYP2A13/2F1-humanized-and-LCN male mice (1.98 \pm 0.33 and $2.12 \pm 0.18 \mathrm{~g}$, respectively). These values were comparable to the values previously reported for 2-month-old male LCN mice ( $2.04 \pm 0.49 \mathrm{~g}$ ) (Gu et al., 2003) but were significantly greater than the liver weights in Cyp2abfgs-null and CYP2A13/2F1-humanized mice $(1.25 \pm 0.12$ and $1.21 \pm 0.11 \mathrm{~g}$, respectively). Similar to what was reported previously for the LCN mice, there was a significant increase in serum alanine aminotransferase (approximately 2-fold) and a decrease in serum cholesterol (approximately 2-fold) in Cyp2abfgs-null-and-LCN and CYP2A13/ 2F1-humanized-and-LCN mice compared with Cyp2abfgs-null and CYP2A13/2F1-humanized mice, respectively (Supplemental Fig. 2, A and B). The serum alkaline phosphatase level was also increased (by approximately 2-fold), but there was no change in serum levels of albumin, blood urea nitrogen, or total bilirubin (Supplemental Fig. 2, C-F).

Liver, Lung, and Nasal Microsomal P450-Mediated NA Bioactivation In Vitro. To assess the relative activity of different organs to metabolize NA in the various mouse strains, we compared the rates of microsomal NA-GSH formation in the liver, lung (whole lung), and olfactory mucosa of CYP2A13/2F1-humanized-and-LCN and Cyp2abfgs-null-and-LCN mice, with NA at a saturating concentration $(100 \mu \mathrm{M})$. As shown in Table 1, lung and liver microsomal activity was significantly lower than in olfactory mucosa $(P<0.0001)$ in both mouse strains, but the difference was much greater in the CYP2A13/2F1humanized-and-LCN mice than in Cyp2abfgs-null-and-LCN mice.

A genotype-dependent difference in the ability to bioactivate NA in vitro was observed in microsomes from the olfactory mucosa and lungs (where the human CYP2A13 and CYP2F1 transgenes were expressed in the CYP2A13/2F1-humanized-and-LCN mice but not in the Cyp2abfgs-null-and-LCN mice) but not in the liver (where the human transgenes were not expressed and $\mathrm{Cpr}$ expression was abolished). Lung microsomes from CYP2A13/2F1-humanizedand-LCN mice had a greater ability to bioactivate NA than Cyp2abfgs-null-and-LCN mice (by 67\%). This genotype-dependent difference was more prominent for microsomal activity of the nasal olfactory mucosa (3.9-fold higher in CYP2A13/2F1-humanized-andLCN than in Cyp2abfgs-null-and-LCN mice). Overall, these data confirm that nasal and lung microsome activity in the CYP2A13/2F1-humanizedand-LCN and Cyp2abfgs-null-and-LCN mice was like that previously found in the CYP2A13/2F1-humanized and Cyp2abfgs-null mice, respectively ( $\mathrm{Li}$ et al., 2017), whereas liver microsome activity of the two new strains was similar to that found in the original LCN mice (Kovalchuk et al., 2017) (Table 1). Thus, nasal and lung microsomal NA oxidation activity (and by inference, transgenic CYP2A13/2F1 expression) was not altered by hepatic CPR status.

NA and NA-GSH Levels in the Plasma of CYP2A13/ 2F1-Humanized-and-LCN Mice and Cyp2abfgs-Null-and-LCN Mice. Levels of NA and NA-GSH were measured in the plasma of CYP2A13/2F1-humanized, Cyp2abfgs-null, CYP2A13/2F1-humanized-and-LCN, and Cyp2abfgs-null-and-LCN mice at multiple time points, starting immediately after termination of a single 4-hour inhalation exposure to $10 \mathrm{ppm}$ NA. NA levels in the plasma of mice with abolished hepatic CPR expression (CYP2A13/2F1-humanizedand-LCN and Cyp2abfgs-null-and-LCN) were significantly higher $(P<0.001)$ compared with corresponding controls with normal hepatic CPR expression (CYP2A13/2F1-humanized and Cyp2abfgs-null) during the first 8 hours after termination of NA exposure (Fig. 2, A and C). Notably, NA levels in plasma continued to increase in CYP2A13/ 2F1-humanized-and-LCN and Cyp2abfgs-null-and-LCN mice after termination of active NA inhalation exposure. The trend of NA elevation was similar between the two mouse strains on the LCN background, although the observed NA elevation during the first 4 hours after NA exposure termination, compared with the 0 -hour time point, was statistically significant only for Cyp2abfgs-null-and-LCN mice $(P<0.05)$. There was also a small, but statistically significant, difference $(P<0.05)$ in NA plasma levels between CYP2A13/2F1-humanized-and-LCN and Cyp2abfgsnull-and-LCN mice at 4 and 8 hours after termination of NA exposure (Fig. 2E). Unlike the relatively steady plasma NA levels during the first 8 hours after NA exposure termination in mice on the LCN background, NA levels in the plasma of mice with normal hepatic CPR expression (CYP2A13/2F1-humanized and Cyp2abfgs-null) declined rapidly during the first 4 hours after termination of NA exposure (Fig. 2, A and C), 
A

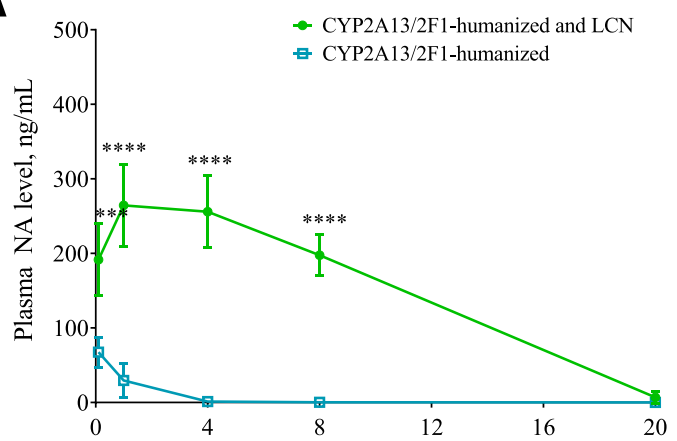

C

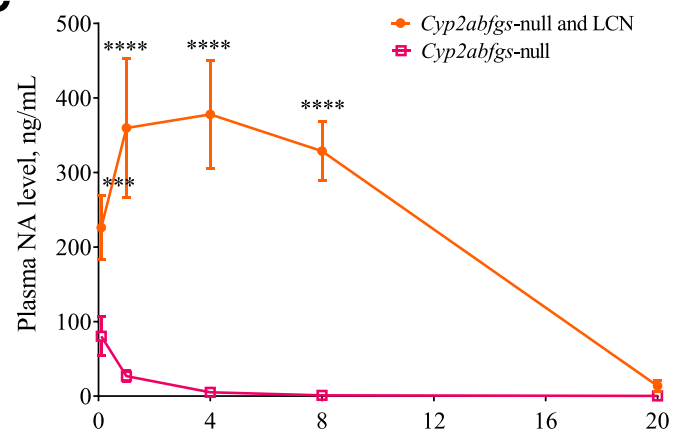

E

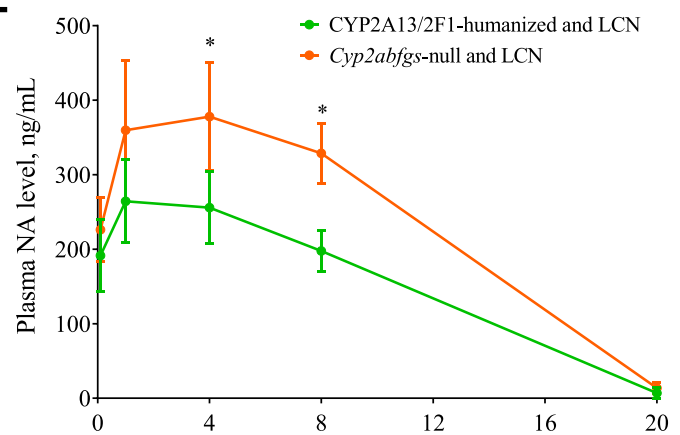

G

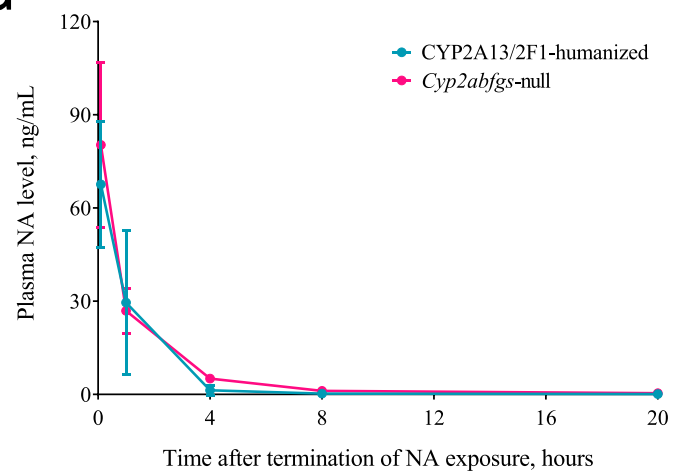

B

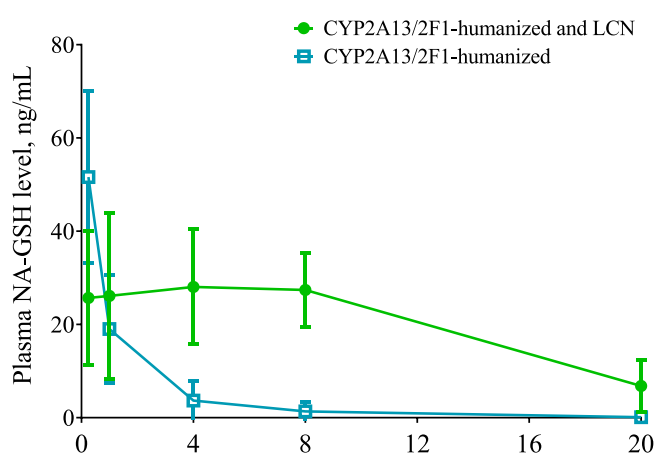

D

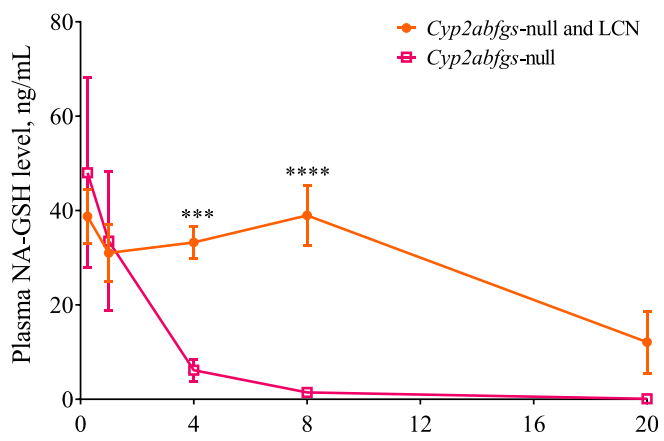

$\mathbf{F}$

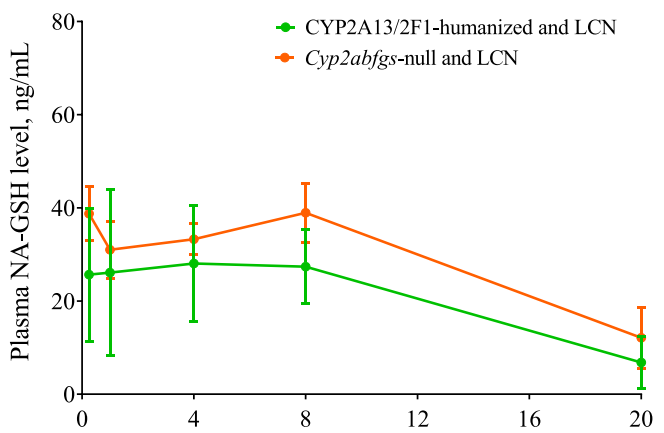

H

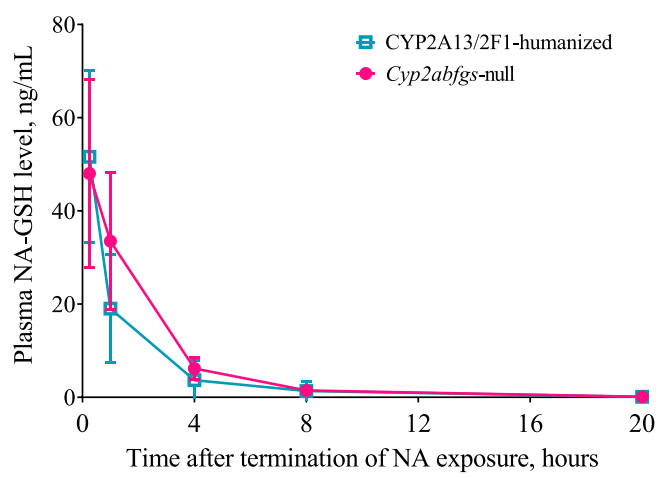

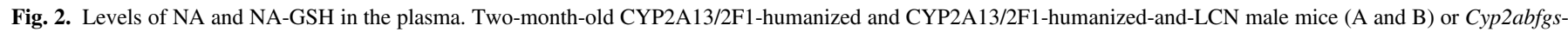

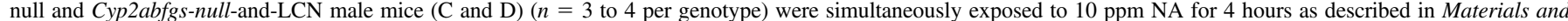

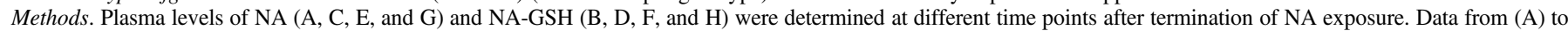

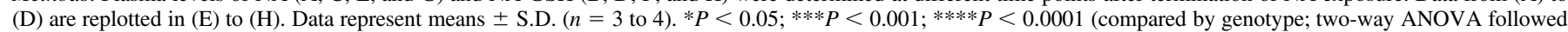
by the Bonferroni test for multiple comparisons).

and there was no difference between CYP2A13/2F1-humanized and Cyp2abfgs-null mice (Fig. 2G).

NA-GSH levels in plasma immediately after termination of NA inhalation exposure were similar among the four different genotype groups, which had either normal or compromised hepatic CPR expression (Fig. 2, B and D). NA-GSH levels declined rapidly in the plasma of CYP2A13/2F1-humanized mice and Cyp2abfgs-null mice, approaching the detection limit ( 0.6 pmol on column) by 8 hours after termination of NA exposure; there was no difference between the two strains (Fig. 2H). In contrast, relatively high and constant levels of NAGSH were maintained in the plasma of CYP2A13/2F1-humanized-andLCN and Cyp2abfgs-null-and-LCN mice during the first 8 hours after 
TABLE 2

Toxicokinetic parameters of plasma NA and NA-GSH in Cyp2abfgs-null, CYP2A13/2F1-humanized, Cyp2abfgs-null-and-LCN, and CYP2A13/ 2F1-humanized-and-LCN mice

Data from Fig. 2 are used for calculation of various toxicokinetic parameters. Results are presented as means \pm S.D. (n $=3$ to 4 per group).

\begin{tabular}{|c|c|c|c|c|c|}
\hline Analyte & Strain & $\mathrm{AUC}_{0-20 \mathrm{~h}}$ & $t_{1 / 2}$ & $\mathrm{CL} / \mathrm{F}$ & $C_{\max }$ \\
\hline & & $\mu g \cdot \min / m l$ & $\min$ & $\mathrm{ml} / \mathrm{min}$ & $n g / m l$ \\
\hline \multirow{4}{*}{ NA } & Cyp2abfgs-null & $4.2 \pm 0.9$ & $3.5 \pm 1.4$ & $72.3 \pm 17.7$ & $80.3 \pm 26.5$ \\
\hline & CYP2A13/2F1-humanized & $4.6 \pm 3.9$ & $3.2 \pm 1.1$ & $122 \pm 78$ & $67.6 \pm 20.2$ \\
\hline & Cyp 2 abfgs-null-and-LCN & $280 \pm 40^{a}$ & $43 \pm 11$ & $1.1 \pm 0.3^{b}$ & $226 \pm 43^{a}$ \\
\hline & CYP2A13/2F1-humanized-and-LCN & $170 \pm 20^{c, d}$ & $57 \pm 39$ & $1.9 \pm 0.2^{c}$ & $192 \pm 49^{c}$ \\
\hline \multirow[t]{4}{*}{ NA-GSH } & Cyp2abfgs-null & $5.2 \pm 1.1$ & N/A & N/A & $48.0 \pm 20.1$ \\
\hline & CYP2A13/2F1-humanized & $3.2 \pm 2.6$ & N/A & N/A & $51.6 \pm 18.4$ \\
\hline & Cyp 2 abfgs-null-and-LCN & $30 \pm 4^{b}$ & N/A & N/A & $38.8 \pm 5.7$ \\
\hline & CYP2A13/2F1-humanized-and-LCN & $26 \pm 8^{e}$ & N/A & N/A & $25.7 \pm 14.3$ \\
\hline
\end{tabular}

\footnotetext{
$\mathrm{CL}$, clearance; N/A, not applicable; $t_{1 / 2}$, elimination half-life.

${ }^{a} P<0.0001$, compared with Cyp2abfgs-null mice.

${ }^{b} P<0.05$, compared with Cyp2abfgs-null mice.

${ }^{c} P<0.0001$, compared with CYP2A13/2F1-humanized mice.

${ }^{d} P<0.001$, compared with Cyp2abfgs-null-and-LCN mice.

${ }^{e} P<0.05$, compared with CYP2A13/2F1-humanized mice.
}

termination of NA exposure (Fig. 2F), which coincided with elevated and steady plasma NA levels during the same postexposure time period (Fig. 2E). In addition, CYP2A13/2F1-humanized-and-LCN mice showed a trend of lower plasma NA-GSH levels compared with Cyp2abfgs-null-and-LCN mice (Fig. 2F).

Toxicokinetic analysis of the data from Fig. 2 (Table 2) revealed approximately 2.8 -fold higher $C_{\max }$ values for NA in mice with compromised hepatic CPR expression than in mice with normal hepatic CPR expression. The AUC values for NA were approximately 36-fold higher in CYP2A13/2F1-humanized-and-LCN than in CYP2A13/ 2F1-humanized mice and approximately 66-fold higher in Cyp2abfgsnull-and-LCN than in Cyp2abfgs-null mice. There was an approximately 1.7-fold increase $(P<0.05)$ in AUC for NA levels in Cyp2abfgs-null-and-LCN mice compared with CYP2A13/2F1humanized-and-LCN mice. The rate of NA clearance was significantly decreased in CYP2A13/2F1-humanized-and-LCN and Cyp2abfgs-null-and-LCN mice (approximately 64-fold and 66-fold, respectively) compared to CYP2A13/2F1-humanized and Cyp2abfgs-null mice, respectively. In addition, the plasma elimination half-life of NA was substantially increased in CYP2A13/2F1-humanized-and-LCN and Cyp2abfgs-null-andLCN mice (by approximately 18 -fold and 12-fold, respectively) over CYP2A13/2F1-humanized and Cyp2abfgs-null mice, respectively. AUC values for NA-GSH were also increased by approximately 7.8 -fold in CYP2A13/2F1-humanized-and-LCN compared with CYP2A13/2F1humanized mice and by approximately 5.7-fold in Cyp2abfgs-null-andLCN compared with Cyp2abfgs-null mice.

NA and NA-GSH Levels in the Lung of CYP2A13/2F1-Humanized-and-LCN Mice and Cyp2abfgs-Null-and-LCN Mice. Levels of NA and NA-GSH were determined in lungs of mice with normal or compromised hepatic CPR expression at different time points after termination of inhalation exposure to $10 \mathrm{ppm}$ NA. The NA level immediately after termination of NA inhalation exposure was 3.9-fold higher in CYP2A13/2F1-humanized-and-LCN mice than in CYP2A13/ 2F1-humanized mice and 3.5-fold higher in Cyp2abfgs-null-and-LCN mice than in Cyp2abfgs-null mice (Fig. 3, A and C). For mice of all four genotypes, NA levels in the lung (Fig. 3) were estimated (by assuming $1 \mathrm{ml}$ plasma to be $1 \mathrm{~g}$ in weight) to be approximately 4-fold to 6-fold higher than in the plasma (Fig. 2) at 0 hours postexposure (see Fig. 4A for a direct comparison). In addition, there was a trend of higher NA levels in the lungs of Cyp2abfgs-null mice than in CYP2A13/2F1humanized mice regardless of their hepatic CPR status $(33 \%$ and
$23 \%$ higher in mice with normal and compromised hepatic CPR expression, respectively) (Fig. 3, E and G). NA levels in the lungs of CYP2A13/2F1-humanized and Cyp2abfgs-null mice decreased rapidly, and they were undetectable at 6 hours after termination of NA exposure (Fig. 3, A and C). In contrast, NA levels in the lungs of mice with compromised hepatic CPR expression decreased slowly and remained relatively high at 6 hours after termination of active NA exposure (Fig. 3E).

NA-GSH levels in the lung were nearly identical in all groups of NA-exposed mice immediately after exposure termination despite a significant difference in NA levels in plasma and tissues between mice with normal and compromised hepatic CPR expression. NA-GSH levels in the lungs decreased significantly $(P<0.01)$ at 2 hours postexposure in mice with normal hepatic CPR expression (Fig. $3 \mathrm{H}$ ), but they were relatively steady in mice with compromised hepatic CPR expression (Fig. 3F). NA-GSH adducts were not detected at 6 hours postexposure in CYP2A13/2F1-humanized and Cyp2abfgs-null mice, in contrast with the CYP2A13/2F1-humanized-and-LCN and Cyp2abfgsnull-and-LCN mice, in which NA-GSH levels became nondetectable only at the 20-hour postexposure time point. For mice of all four genotypes, NA-GSH levels were estimated (by assuming $1 \mathrm{ml}$ plasma to be $1 \mathrm{~g}$ in weight) to be higher in the lung (Fig. 3) than in the plasma (Fig. 2) at 0 hours postexposure (see Fig. 4B for a direct comparison).

NA and NA-GSH Levels in the Liver of CYP2A13/2F1-Humanized-and-LCN Mice and Cyp2abfgs-Null-and-LCN Mice. Hepatic NA levels were 20-fold higher in CYP2A13/2F1-humanized-and-LCN mice than in CYP2A13/2F1-humanized mice and 27-fold higher in Cyp2abfgs-null-and-LCN mice than in Cyp2abfgs-null mice immediately after termination of a 4-hour inhalation exposure (10 ppm) (Fig. 5, $\mathrm{A}$ and C). Moreover, NA levels appeared to be higher (by $33 \%, P=$ 0.0632) in the liver of Cyp2abfgs-null-and-LCN mice, compared with CYP2A13/2F1-humanized-and-LCN mice (Fig. 5E), at 0 hours postexposure. NA levels were somewhat lower in the liver (Fig. 5) than in the lungs (Fig. 3) of mice with normal hepatic CPR expression (by $40 \%$ in Cyp2abfgs-null mice and $20 \%$ in CYP2A13/2F1-humanized mice) immediately after the inhalation exposure, but they were substantially higher in the liver than in the lung of Cyp2abfgs-null-and-LCN (by 4.5-fold) and CYP2A13/2F1-humanized-and-LCN mice (by 3.4-fold) (see Fig. 6A for a direct comparison). NA levels in the liver of mice with normal hepatic CPR expression declined rapidly after termination of active NA exposure, and they were undetectable 6 hours after termination of NA exposure (Fig. 5G). In contrast, relatively steady 

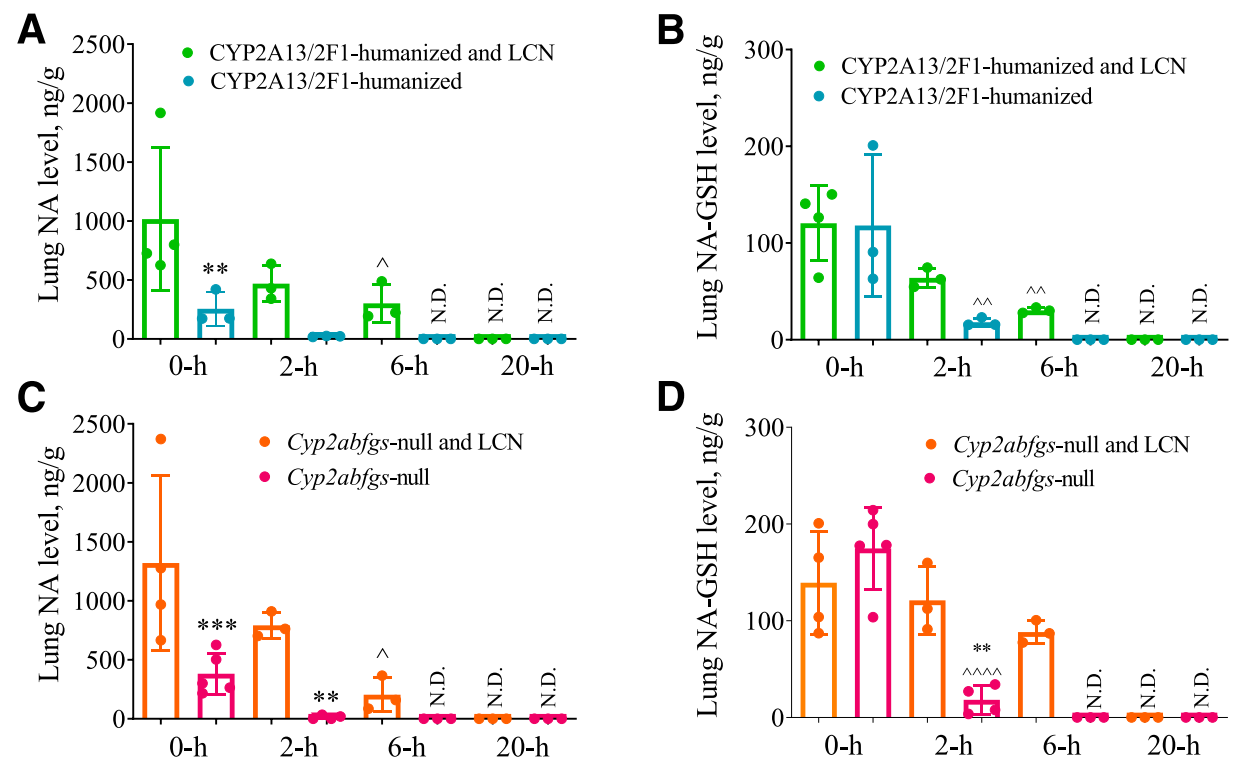

Fig. 3. Levels of NA and NA-GSH in the lung. Two-month-old CYP2A13/2F1-humanized and CYP2A13/2F1-humanized-and-LCN male mice (A and B) or Cyp2abfgs-null and Cyp2abfgsnull-and-LCN male mice (C and D) $(n=3-5$ per genotype for each time point) were exposed to 10 ppm NA for 4 hours. Lungs were collected 0,2 , 6 , or 20 hours after termination of NA exposure.

F
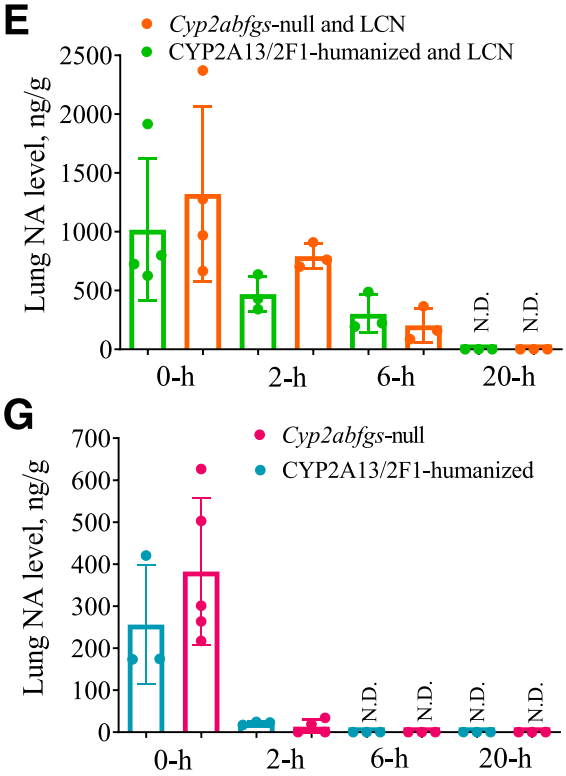

Time after NA exposure termination, hours

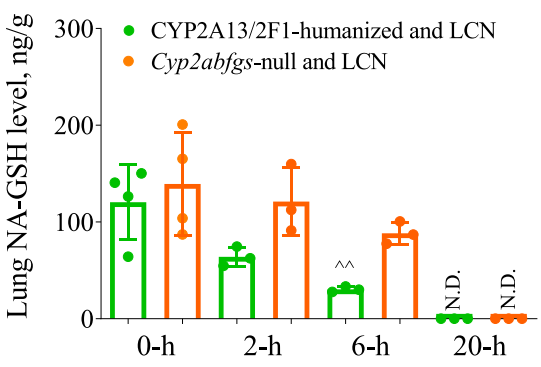

H

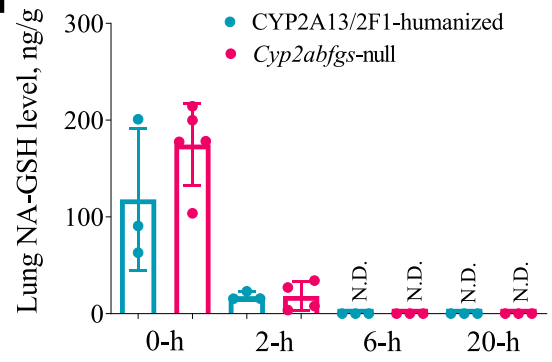

Time after NA exposure termination, hours
NA levels were found in mice with compromised hepatic CPR expression during the first 6 hours of postexposure time, with a trend of higher NA levels in Cyp2abfgs-null-and-LCN mice (Fig. 5E).

Levels of hepatic NA-GSH were approximately 2.5 -fold higher in Cyp2abfgs-null mice than in Cyp2abfgs-null-and-LCN mice and approximately 2.6-fold higher in CYP2A13/2F1-humanized mice than in CYP2A13/2F1-humanized-and-LCN mice at 0 hours postexposure (Fig. 5, B and D). NA-GSH levels decreased more rapidly in mice with normal hepatic CPR expression (Fig. 5H) than in mice with abolished hepatic CPR expression (Fig. 5F), resulting in the disappearance of CPR genotype-related differences by the 2-hour time point and drastically lower NA-GSH levels in mice with normal hepatic CPR expression by the 6-hour time point. However, the adduct levels were not notably different between CYP2A13/2F1humanized-and-LCN and Cyp2abfgs-null-and-LCN mice (Fig. 5F) or between CYP2A13/2F1-humanized and Cyp2abfgs-null mice (Fig. $5 \mathrm{H}$ ). For mice of all four genotypes, NA-GSH levels were higher in the liver (Fig. 5) than in the lung (Fig. 3) at 0 hours postexposure (see Fig. 6B for a direct comparison).

\section{Discussion}

The extent of xenobiotic toxicity in a portal-of-entry, extrahepatic organ is influenced by many factors, ranging from environmental exposure, absorption, and systemic exposure, to metabolic disposition via the target organ and the liver and toxic metabolite generation either locally or systemically. In this study, we examined NA disposition and bioactivation in mouse models undergoing genetic modulation of systemic metabolism of NA by the CYP2A, 2B, 2F, 2G, and $2 \mathrm{~S}$ enzymes of hepatic metabolism by all microsomal P450 enzymes (LCN) and/or of expression of two human enzymes (CYP2A13 and 2F1) active toward NA metabolism in the respiratory tract.

The toxicokinetics of xenobiotics in an inhalation exposure model needs to be analyzed in two different time segments: during and after 

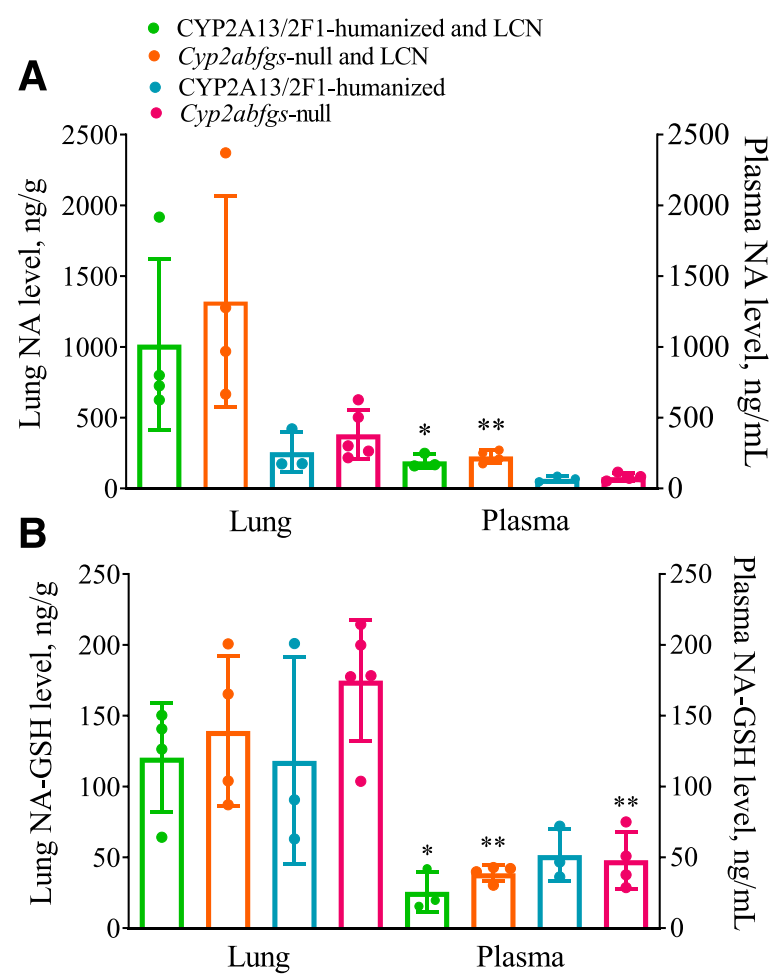

Fig. 4. Comparison of NA and NA-GSH levels in lung and plasma at 0 hours postexposure. Data from Figs. 2 and 3 are replotted for a direct comparison between lung and plasma. Data represent means \pm S.D. $(n=3-5)$. $* P<0.05 ; * * P<0.01$ (compared between lung and plasma for the corresponding genotype; two-way ANOVA followed by Bonferroni's test for multiple comparisons).

active inhalation exposure. During active inhalation exposure, absorption from the airways to the lung and blood is the dominant event but a steady-state plasma level is presumably established eventually, which reflects the sum of contributions by all absorption, distribution, metabolism, and excretion events occurring in the body. This level can be approximated by the level detected at the termination of active inhalation exposure (e.g., the 0-hour data point in Fig. 2). Once inhalation exposure has been terminated, disposition via metabolism and excretion is the driving force that causes plasma and tissue levels to decrease, which includes evaporation from the airway surface for volatile compounds like NA as well as metabolism of circulating NA by $\mathrm{P} 450$ enzymes in the liver and extrahepatic organs. This postexposure "decay" was rapid for NA in mice with normal hepatic P450 activity, as reported previously in other mouse models (Li et al., 2011; Kovalchuk et al., 2017), but was much slower in mice with liver-specific loss of CPR expression (Fig. 2, A and C). Furthermore, during active exposure, the steady-state NA levels in the liver, lung, and plasma were also greatly elevated in mice with deficient liver P450 activity, presumably because of the decreased rate of metabolic disposition of circulating NA in the liver.

Interestingly, although NA levels in the liver and lung decreased with postexposure time, plasma levels of NA continued to increase after the termination of inhalation exposure in mice with deficient hepatic $\mathrm{P} 450$ activity (Fig. 2, A and C), indicating redistribution of stored NA from other sites within the body to blood. Given that NA levels in the liver are much higher than in the lung and plasma (Figs. 4 and 6) and NA has the ability to diffuse through membranes, it can be deduced that the liver and possibly other extra pulmonary tissues not examined here (e.g., adipose tissue) are the major source of this postexposure increase in plasma NA levels and the main cause for the prolonged occurrence of NA exposure and bioactivation in the lung after (nose-only) inhalation exposures have ceased. This ability of inhaled NA to accumulate in the liver at high levels during active exposure and then be slowly released to the circulation and the target tissue for toxicity (lung) over many hours (Figs. 2 and 5), in cases where hepatic P450 activity is suppressed, has important implications for predicting risks of lung toxicity from NA exposure in individuals with compromised hepatic P450 function. In that regard, hepatic P450 activity toward xenobiotic metabolism can be suppressed not only by $\mathrm{P} 450$ genetic polymorphism or drug-xenobiotic interactions but also by various liver diseases (George et al., 1995; Frye et al., 2006)

Our data further suggested that increases in NA circulating or tissue levels during active inhalation exposure to NA did not have a significant impact on the extent of target tissue NA bioactivation in the lung. NA-GSH levels in the lungs of Cyp2abfgs-null or CYP2A13/2F1humanized mice were the same regardless of whether liver $\mathrm{P} 450$ was functional (Fig. 3, B and D) and despite significant differences in plasma (Fig. 2, A and C) or lung NA levels (Fig. 3, A and C) at the termination of active NA exposure. This intriguing observation may be explained by saturation of the lung P450 enzymes by NA, which might be at very high levels in the airway and alveolar epithelial cells, during active exposure (10 ppm). However, the impact of circulating and tissue levels of NA on the extent of NA bioactivation in the lung during the postexposure period was obvious, given the LCN genotype-related differences in tissue NA-GSH levels at 2 and 6 hours after termination of NA exposure (Fig. 3, B and D). It remains to be determined whether the same cells or lung regions are involved in NA metabolism during both active exposure and postexposure periods, as it is not clear whether circulating NA and airborne NA would be absorbed by lung tissue at differing sites.

It should also be noted that in the LCN mice with intact CYP2ABFGS enzymes in the lung, increases in circulating or tissue NA levels during active inhalation exposure to NA were accompanied by a significant increase in the extent of target tissue NA bioactivation in the lung (Kovalchuk et al., 2017). This earlier finding suggested that the lung P450 enzymes were not saturated by NA. The apparent discrepancy on effects of LCN status on WT (Kovalchuk et al., 2017) or Cyp2abfgs-null background (this study) is most likely because of much lower lung tissue NA levels achieved in the LCN mice than in Cyp2abfgs-null-and-LCN mice, although differences in enzyme kinetic properties between CYP2ABFGS enzymes (more efficient toward NA) and other P450 enzymes in the lung may also be relevant.

Our data also show that transgenic CYP2A13/2F1 can contribute to systemic disposition of circulating NA in humanized mice with compromised hepatic P450 function (Fig. 2E) but not in mice with normal hepatic P450 activity (Fig. 2G). It should be noted that the source of this extrahepatic contribution is most likely nasal mucosa, where the human transgene expression level (Wei et al., 2012) and NA metabolism activity (Table 1) were much higher than in the lung. A dominant nasal (but not lung) contribution to NA disposition during the postexposure period would also explain the corresponding decreases in NA-GSH levels in the lung (Fig. 3F). In that connection, the persistence of in vivo NA-GSH formation in the lungs of Cyp2abfgs-null-and-LCN mice (Fig. 3D) demonstrated the ability of non-CYP2ABFGS enzymes of the lung to bioactivate NA in mice with deficient hepatic NA metabolism. Overall, the extrahepatic contributions to NA disposition were relatively small and were insufficient to change circulating NA levels during active NA exposure (Fig. 2E, 0 hours) or to cause significant changes in NA levels in the lung (Fig. 3E), contrary to the effects of LCN status (Figs. 2, $\mathrm{A}$ and $\mathrm{C}$, and $3, \mathrm{~A}$ and $\mathrm{C}$ ).

The results of our recent study on LCN mice (Kovalchuk et al., 2017) and of this study on mouse models that are "humanized" by having a much lower overall rate of NA metabolism in the lung (relative to WT 
A

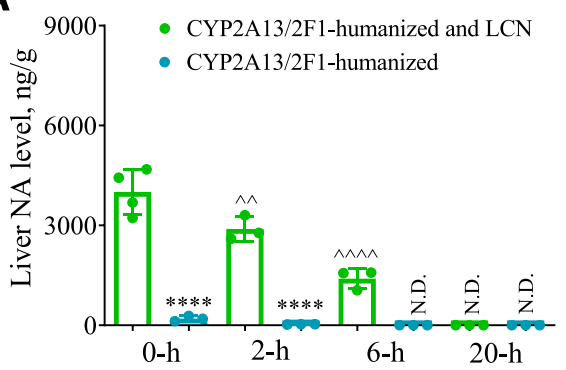

C

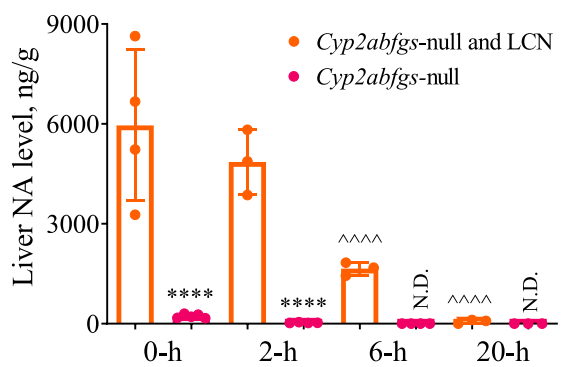

E

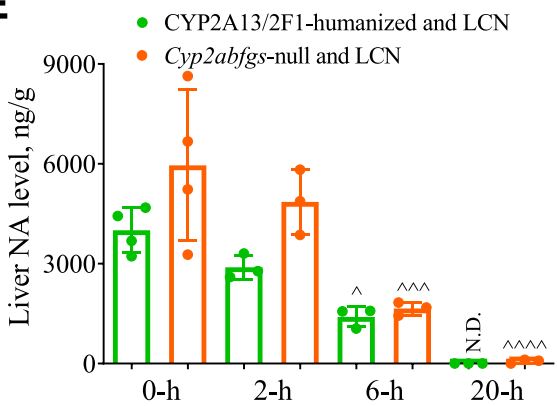

G

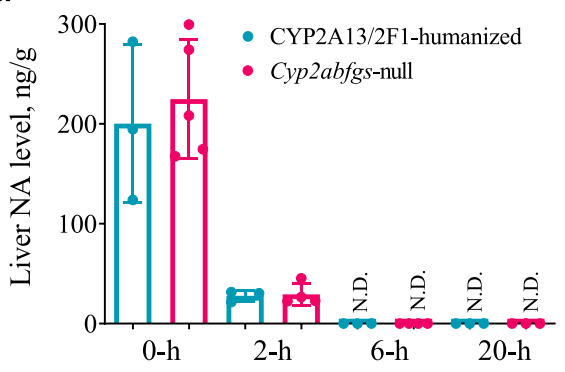

Time after NA exposure termination, hours
B

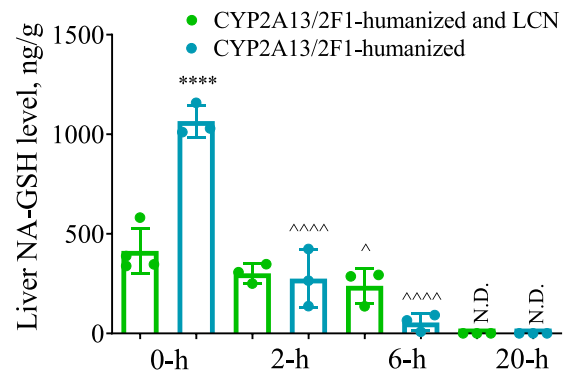

D

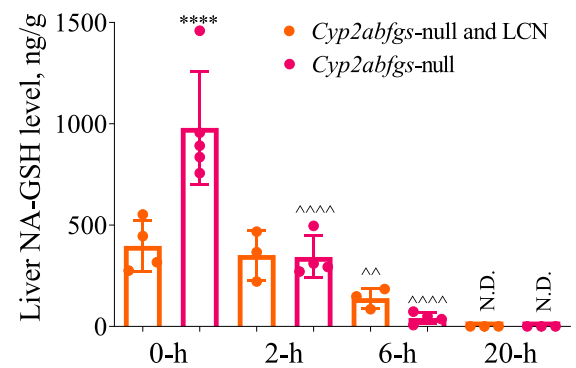

F

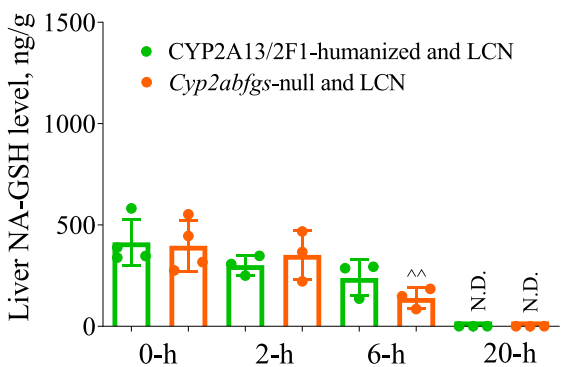

H

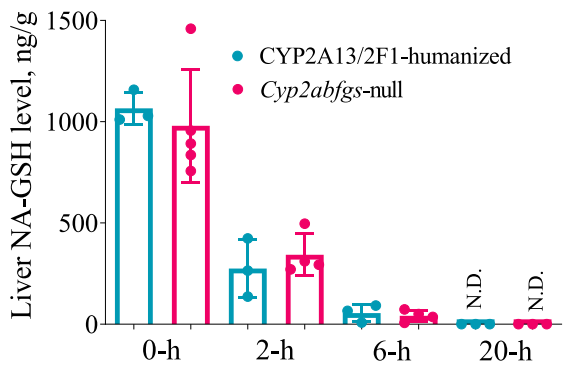

Time after NA exposure termination, hours
Fig. 5. Levels of NA and NA-GSH in the liver. Two-month-old CYP2A13/2F1-humanized and CYP2A13/2F1-humanized-and-LCN male mice (A) and Cyp2abfgs-null and Cyp2abfgs-null-and-LCN male mice (B) $(n=3-5$ per genotype for each time point $)$ were exposed to $10 \mathrm{ppm}$ NA for 4 hours. Livers from the same groups of mice described in Fig. 3 were collected for determination of NA (A, C, E, and G) and NA-GSH $(\mathrm{B}, \mathrm{D}, \mathrm{F}$, and $\mathrm{H})$ in tissue homogenates. Data from (A) to (D) are replotted in (E) to (H). Data represent means \pm S.D. $(n=3-5)$. $* * * * P<0.0001$; compared between genotypes for the corresponding time point. $\wedge P<$ $0.05 ; \wedge \wedge P<0.01 ; \wedge \wedge \wedge \mathrm{P}<0.001 ; \wedge \wedge \wedge \wedge P<$ 0.0001 (compared with 0 hours, for the corresponding genotype; two-way ANOVA followed by Bonferroni's test for multiple comparisons). mice) and by expressing human CYP2A13 and CYP2F1 strongly support a protective role of hepatic $\mathrm{P} 450$ enzymes in reducing the tissue burden and extent of bioactivation of NA in the lung, at least during the postexposure period. However, contrary to the expected protective role, LCN mice showed lower sensitivity to NA-induced lung toxicity than WT mice after inhalation exposure to NA at occupationally relevant concentrations; this finding suggested that liver P450-generated NA metabolites also contribute to toxicity in lung airways (Kovalchuk et al., 2017). Thus, it remains to be determined whether liver-produced reactive NA metabolites (e.g., NA-oxide and NA-quinones) can be transported to the lung, and whether deficiency of P450-mediated NA metabolism in the liver leads to decreases in the amount of these metabolites in the circulation and lung tissue. A more complete account of NA tissue burden and in situ bioactivation in the lung, as well as the burden of systemically derived reactive NA metabolites, during and after
NA inhalation exposure will facilitate establishment of accurate predictive models for risk assessment in humans.

In conclusion, this study shows that a deficiency in hepatic NA metabolism not only decreases NA systemic disposition but also causes NA to accumulate in tissues after inhalation exposure. Subsequent NA release from tissue is partly responsible for the prolonged occurrence of NA exposure and bioactivation in the lung after inhalation exposures have ceased. Our data also reveal the ability of respiratory tract P450 enzymes to influence the rate of systemic NA disposition in mice with deficient hepatic P450 activity and support a dominant effect of the target tissue bioavailability of NA on the extent of NA bioactivation in the lung. Furthermore, we demonstrate the ability of non-CYP2ABFGS enzymes of the lung to bioactivate NA in mice with deficient hepatic NA metabolism. Taken together, these results support the notion that assessment of toxic potential for NA and other inhaled xenobiotics in 


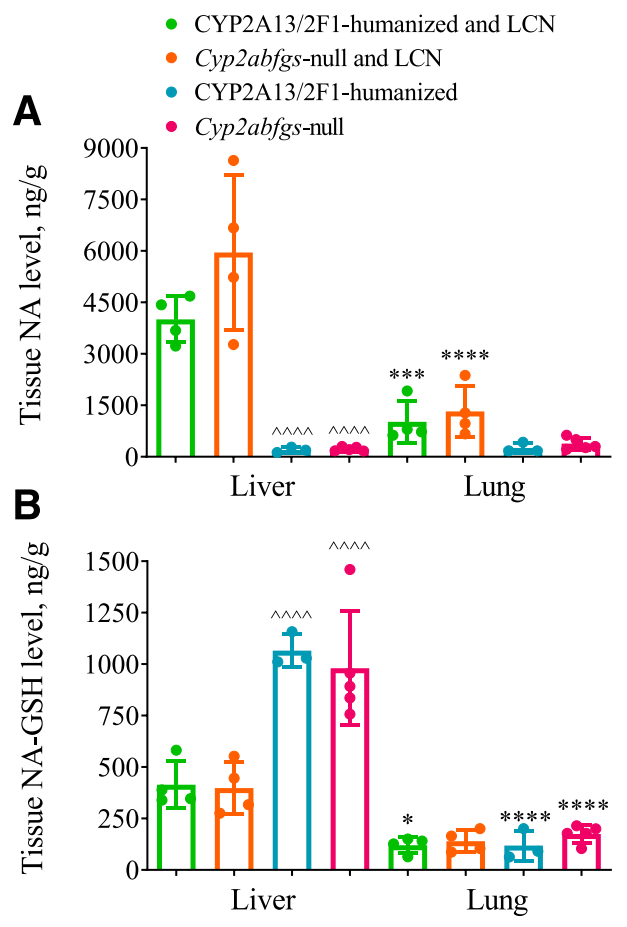

Fig. 6. Comparison of NA and NA-GSH levels in liver and lung at 0 hours postexposure. Data from Figs. 3 and 5 are replotted for a direct comparison between liver and lung. Data represent means \pm S.D. $(n=3-5) . * P<0.05$; $* * * P<0.001$; $* * * * P<0.0001$ (compared between liver and lung for the corresponding genotype) $\wedge \wedge \wedge \wedge P<0.0001$ (compared between CYP2A13/2F1-humanized or Cyp2abfgs-null mice and corresponding LCN mice; two-way ANOVA followed by Bonferroni's test for multiple comparisons).

human lungs should consider the balance between hepatic and lung P450 roles in both bioactivation and disposition, and they suggest potentially large effects of deficiencies in hepatic P450 activity on NA tissue burden and bioactivation in human lungs.

\section{Acknowledgments}

We thank Drs. Alan Buckpitt and Dexter Morin (University of California, Davis) for providing NA-GSH standards. We also acknowledge Weizhu Yang for assistance with mouse breeding.

\section{Authorship Contributions}

Participated in research design: Kovalchuk, Zhang, Kelty, Van Winkle, Ding.

Conducted experiments: Kovalchuk, Kelty.

Performed data analysis: Kovalchuk, Kelty, Van Winkle, Ding.

Wrote or contributed to the writing of the manuscript: Kovalchuk, Zhang, Kelty, Van Winkle, Ding.

\section{References}

Abdo KM, Eustis S, McDonald M, Jokinen M, Adkins B, and Haseman J (1992) Naphthalene: a respiratory tract toxicant and carcinogen for mice. Inhal Toxicol 4:393-409.

Abdo KM, Grumbein S, Chou BJ, and Herbert R (2001) Toxicity and carcinogenicity study in F344 rats following 2 years of whole-body exposure to naphthalene vapors. Inhal Toxicol $\mathbf{1 3}$ 931-950.

Buckpitt A, Boland B, Isbell M, Morin D, Shultz M, Baldwin R, Chan K, Karlsson A, Lin C, Taff A, et al. (2002) Naphthalene-induced respiratory tract toxicity: metabolic mechanisms of toxicity. Drug Metab Rev 34:791-820.

Carr BA, Wan J, Hines RN, and Yost GS (2003) Characterization of the human lung CYP2F1 gene and identification of a novel lung-specific binding motif. J Biol Chem 278:15473-15483.

Chao YC, Kupper LL, Serdar B, Egeghy PP, Rappaport SM, and Nylander-French LA (2006) Dermal exposure to jet fuel JP-8 significantly contributes to the production of urinary naphthols in fuel-cell maintenance workers. Environ Health Perspect 114:182-185.
Cho TM, Rose RL, and Hodgson E (2006) In vitro metabolism of naphthalene by human liver microsomal cytochrome P450 enzymes. Drug Metab Dispos 34:176-183.

Frye RF, Zgheib NK, Matzke GR, Chaves-Gnecco D, Rabinovitz M, Shaikh OS, and Branch RA (2006) Liver disease selectively modulates cytochrome P450-mediated metabolism. Clin Pharmacol Ther 80:235-245.

Genter MB, Marlowe J, Kevin Kerzee J, Dragin N, Puga A, Dalton TP, and Nebert DW (2006) Naphthalene toxicity in mice and aryl hydrocarbon receptor-mediated CYPs. Biochem Biophys Res Commun 348:120-123.

George J, Murray M, Byth K, and Farrell GC (1995) Differential alterations of cytochrome P450 proteins in livers from patients with severe chronic liver disease. Hepatology 21:120-128.

Griego FY, Bogen KT, Price PS, and Weed DL (2008) Exposure, epidemiology and human cancer incidence of naphthalene. Regul Toxicol Pharmacol 51 (Suppl):S22-S26.

Gu J, Weng Y, Zhang QY, Cui H, Behr M, Wu L, Yang W, Zhang L, and Ding X (2003) Liverspecific deletion of the NADPH-cytochrome P450 reductase gene: impact on plasma cholesterol homeostasis and the function and regulation of microsomal cytochrome P450 and heme oxygenase. J Biol Chem 278:25895-25901.

Hu J, Sheng L, Li L, Zhou X, Xie F, D'Agostino J, Li Y, and Ding X (2014) Essential role of the cytochrome P450 enzyme CYP2A5 in olfactory mucosal toxicity of naphthalene. Drug Metab Dispos 42:23-27.

Kakareka SV and Kukharchyk TI (2003) PAH emission from the open burning of agricultural debris. Sci Total Environ 308:257-261.

Kovalchuk N, Kelty J, Li L, Hartog M, Zhang QY, Edwards P, Van Winkle L, and Ding X (2017) Impact of hepatic P450-mediated biotransformation on the disposition and respiratory tract toxicity of inhaled naphthalene. Toxicol Appl Pharmacol 329:1-8.

Li L, Carratt S, Hartog M, Kovalchik N, Jia K, Wang Y, Zhang QY, Edwards P, Winkle LV, and Ding X (2017) Human CYP2A13 and CYP2F1 mediate naphthalene toxicity in the lung and nasal mucosa of CYP2A13/2F1-humanized mice. Environ Health Perspect 125:67004.

Li L, Megaraj V, Wei Y, and Ding X (2014) Identification of cytochrome P450 enzymes critical for lung tumorigenesis by the tobacco-specific carcinogen 4-(methylnitrosamino)-1-(3-pyridyl)-1butanone (NNK): insights from a novel Cyp2abfgs-null mouse. Carcinogenesis 35:2584-2591.

Li L, Wei Y, Van Winkle L, Zhang QY, Zhou X, Hu J, Xie F, Kluetzman K, and Ding X (2011) Generation and characterization of a Cyp2f2-null mouse and studies on the role of CYP2F2 in naphthalene-induced toxicity in the lung and nasal olfactory mucosa. J Pharmacol Exp Ther 339:62-71.

McClean MD, Osborn LV, Snawder JE, Olsen LD, Kriech AJ, Sjödin A, Li Z, Smith JP, Sammons DL, Herrick RF, et al. (2012) Using urinary biomarkers of polycyclic aromatic compound exposure to guide exposure-reduction strategies among asphalt paving workers. Ann Occup Hyg 56:1013-1024

Merchant-Borna K, Rodrigues EG, Smith KW, Proctor SP, and McClean MD (2012) Characterization of inhalation exposure to jet fuel among U.S. Air Force personnel. Ann Occup Hyg 56: 736-745.

Preuss R, Angerer J, and Drexler H (2003) Naphthalene-an environmental and occupational toxicant. Int Arch Occup Environ Health 76:556-576.

Richieri PR and Buckpitt AR (1987) Efflux of naphthalene oxide and reactive naphthalene metabolites from isolated hepatocytes. J Pharmacol Exp Ther 242:485-492.

Serdar B, Egeghy PP, Gibson R, and Rappaport SM (2004) Dose-dependent production of urinary naphthols among workers exposed to jet fuel (JP-8). Am J Ind Med 46:234-244.

Shimada T, Takenaka S, Kakimoto K, Murayama N, Lim YR, Kim D, Foroozesh MK, Yamazaki H, Guengerich FP, and Komori M (2016) Structure-function studies of naphthalene, phenanthrene, biphenyl, and their derivatives in interaction with and oxidation by cytochromes P450 $2 \mathrm{~A} 13$ and 2A6. Chem Res Toxicol 29:1029-1040.

Shultz MA, Choudary PV, and Buckpitt AR (1999) Role of murine cytochrome P-450 2F2 in metabolic activation of naphthalene and metabolism of other xenobiotics. J Pharmacol Exp Ther 290:281-288.

Su T, Bao Z, Zhang QY, Smith TJ, Hong JY, and Ding X (2000) Human cytochrome P450 CYP2A13: predominant expression in the respiratory tract and its high efficiency metabolic activation of a tobacco-specific carcinogen, 4-(methylnitrosamino)-1-(3-pyridyl)-1-butanone. Cancer Res 60:5074-5079.

Tingle MD, Pirmohamed M, Templeton E, Wilson AS, Madden S, Kitteringham NR, and Park BK (1993) An investigation of the formation of cytotoxic, genotoxic, protein-reactive and stable metabolites from naphthalene by human liver microsomes. Biochem Pharmacol 46:1529-1538.

Verschoyle RD, Martin J, and Dinsdale D (1997) Selective inhibition and induction of CYP activity discriminates between the isoforms responsible for the activation of butylated hydroxytoluene and naphthalene in mouse lung. Xenobiotica 27:853-864

Warren DL, Brown DL Jr, and Buckpitt AR (1982) Evidence for cytochrome P-450 mediated metabolism in the bronchiolar damage by naphthalene. Chem Biol Interact 40:287-303.

Weems JM and Yost GS (2010) 3-Methylindole metabolites induce lung CYP1A1 and CYP2F1 enzymes by AhR and non-AhR mechanisms, respectively. Chem Res Toxicol 23:696-704.

Wei Y, Wu H, Li L, Liu Z, Zhou X, Zhang QY, Weng Y, D'Agostino J, Ling G, Zhang X, et al (2012) Generation and characterization of a CYP2A13/2B6/2F1-transgenic mouse model. Drug Metab Dispos 40:1144-1150.

Wilson AS, Davis CD, Williams DP, Buckpitt AR, Pirmohamed M, and Park BK (1996) Characterisation of the toxic metabolite(s) of naphthalene. Toxicology 114:233-242.

Witschi H, Espiritu I, Maronpot RR, Pinkerton KE, and Jones AD (1997) The carcinogenic potential of the gas phase of environmental tobacco smoke. Carcinogenesis 18:2035-2042.

Wu L, Gu J, Weng Y, Kluetzman K, Swiatek P, Behr M, Zhang QY, Zhuo X, Xie Q, and Ding X (2003) Conditional knockout of the mouse NADPH-cytochrome p450 reductase gene. Genesis 36:177-181.

Address correspondence to: Dr. Xinxin Ding, Department of Pharmacology and Toxicology, College of Pharmacy, University of Arizona, Tucson, AZ 85721. E-mail: xding@pharmacy.arizona.edu; or Dr. Laura Van Winkle, Department of Anatomy Physiology and Cell Biology, School of Veterinary Medicine, University of California, Davis, CA 95616. E-mail: Isvanwinkle@ucdavis.edu 